\title{
The Köthe Dual of an Abstract Banach Lattice
}

\author{
E. Jiménez Fernández, ${ }^{1}$ M. A. Juan, ${ }^{2}$ and E. A. Sánchez-Pérez ${ }^{2}$ \\ ${ }^{1}$ Departamento de Análisis Matemático, Facultad de Ciencias, Universidad de Granada, Campus Fuentenueva S/N, \\ 18071 Granada, Spain \\ ${ }^{2}$ Instituto Universitario de Matemática Pura y Aplicada, Universidad Politécnica de Valencia, Camino de Vera S/N, \\ 46022 Valencia, Spain
}

Correspondence should be addressed to E. Jiménez Fernández; edjimfer@mat.upv.es

Received 28 May 2013; Accepted 6 June 2013

Academic Editor: Miguel Martin

Copyright (C) 2013 E. Jiménez Fernández et al. This is an open access article distributed under the Creative Commons Attribution License, which permits unrestricted use, distribution, and reproduction in any medium, provided the original work is properly cited.

We analyze a suitable definition of Köthe dual for spaces of integrable functions with respect to vector measures defined on $\delta$-rings. This family represents a broad class of Banach lattices, and nowadays it seems to be the biggest class of spaces supported by integral structures, that is, the largest class in which an integral representation of some elements of the dual makes sense. In order to check the appropriateness of our definition, we analyze how far the coincidence of the Köthe dual with the topological dual is preserved.

\section{Introduction}

The aim of this paper is to extend the usual notion of Köthe dual of a Banach function space over a $\sigma$-finite measure to the general class of Banach lattices. Recall that the Köthe dual or associate space of a Banach function space $X$ over a $\sigma$-finite measure $\mu$ is defined by the set of all the functionals belonging to the topological dual $X^{*}$ that can be represented as integrals; that is, if $\varphi \in X^{*}$ is such a functional, there is a measurable function $g_{\varphi}$ such that $f \leadsto \int g_{\varphi} f d \mu$ equals $\varphi(f)$ for all $f \in X$.

Integration with respect to Banach space valued vector measures on $\delta$-rings is at the basis of a fruitful representation technique for abstract lattices that has been recently developed. Certainly, the spaces $L^{1}(\nu)$ of integrable functions and $L_{w}^{1}(\nu)$ of weakly integrable functions represent a large family of Banach lattices. Nowadays it is well known that each order continuous Banach lattice can be written (isometrically and in order) as an $L^{1}(\nu)$-space of a certain vector measure $v$ on a $\delta$-ring, and an equivalent result holds for Banach lattices with the Fatou property and some additional requirements with the spaces $L_{w}^{1}(\nu)$ (see [1, Theorems 4 and 8$]$ and [2]; see also [3, pp. 22-23]). Similar results follow also under the assumption of $p$-convexity of the Banach lattices, using in this case spaces of $p$-integrable functions (see [4-6]).

Far from being a formal requirement, the use of vector measures on $\delta$-rings is essential for our purposes. The reason is that this is the way of extending the classical representation results-that hold for Banach function spaces based on $\sigma$ finite measure spaces-to the abstract case, that include for instance representations of spaces $\ell^{p}(\Gamma)$ for an uncountable set of indexes $\Gamma$ based on an integration structure. On the other hand, it must be said that the representation for the $\sigma$ finite case is completely covered by the integration structure provided by vector measures on $\sigma$-algebras. Regarding spaces of integrable functions with respect to such vector measures, several descriptions of the dual space of an $L^{1}$-space (an $L^{p_{-}}$ space) of a vector measure are nowadays known (see [710]). Notice that, since the spaces are order continuous, these results give directly a description of the corresponding dual spaces.

In this paper we propose a concrete definition of Köthe dual space for a general class of Banach lattices. Our purpose includes two natural properties that one can expect for such a space; that is,

(1) its elements must be-at least locally-integrals, that is, functionals defined by integrable functions, and

(2) the set of such elements (the Köthe dual) coincides with the whole topological dual whenever the original lattice is order continuous.

As the reader may notice, we will give an easy description of such a space which covers the hole existing in 
the mathematical literature regarding the notion of the space of the integrals as elements of the dual space.

\section{Preliminaries}

2.1. Banach Lattices. We recall in this section the notions concerning Banach lattices required for a suitable reading of the paper.

Let $E$ be a Banach lattice with order $\leq$ and norm $\|\cdot\|$. A linear subspace $F$ of $E$ is called an ideal of $E$ if $x \in F,|y| \leq|x|$ implies $y \in F$. An ideal $F$ in $E$ is said to be super order dense in $E$ if for every $0 \leq x \in E$ there exists an increasing sequence $\left(x_{n}\right)_{n} \subset F$ so that $0 \leq x_{n} \uparrow x$ in $E$. If this property holds by means of upwards directed systems, $F$ is said to be order dense in $E$. An element $0 \leq e \in E$ such that $x \wedge e=0$ implies $x=0$ is said to be a weak unit of $E$.

The Banach lattice $E$ is said to have $\sigma$-order continuous norm, or briefly, to be $\sigma$-order continuous if for every decreasing sequence $\left(x_{n}\right)_{n} \downarrow 0$ it follows that $\left\|x_{n}\right\| \downarrow 0$. Similarly, $E$ is said to have order continuous norm, or briefly, to be order continuous if this property holds for downwards directed systems. We denote by $E_{a}$ the $\sigma$-order continuous part of $E$, that is, the largest $\sigma$-order continuous ideal in $E$. We will use $E_{a n}$ for the order continuous part of $E$ which in this case is the largest order continuous ideal in $E$.

Finally, the Banach lattice $E$ is said to have the $\sigma$-Fatou property if for every increasing sequence $0 \leq x_{n} \uparrow$ in $E$ with $\sup _{n}\left\|x_{n}\right\|<\infty$, it follows that there exists $x:=\sup _{n} x_{n}$ in $E$ and $\|x\|=\sup _{n \geq 1}\left\|x_{n}\right\|$. Again, $E$ is said to have the Fatou property if this is the case for upwards directed systems.

An operator $T: E \rightarrow F$ between Banach lattices is said to be an order isometry if it is a linear isometry which is also an order isomorphism (i.e., $T$ is also one to one, onto, $\|T x\|_{F}=$ $\|x\|_{E}$ for all $x \in E$ and $T(x \wedge y)=T x \wedge T y$ for all $\left.x, y \in E\right)$. In this case $E$ and $F$ are said to be order isometric.

For these other issues related to Banach lattices, see for instance [11-15].

2.2. Integration with respect to Vector Measures on $\delta$-Rings. We recall here the integration theory with respect to vector measures introduced by Lewis in the context of $\sigma$-algebras [16] and extended by Masani and Niemi and Stefansson in the context of $\delta$-rings [17-19]. We refer also to $[2,20]$.

Given $\mathscr{R}$ a $\delta$-ring of subsets of an abstract set $\Omega$, that is, a ring of sets of $\Omega$ closed under countable intersections, we can consider $\mathscr{R}^{\text {loc }}$ the associated $\sigma$-algebra to $\mathscr{R}$ defined by $\mathscr{R}^{\text {loc }}=\{B \subset \Omega: B \cap A \in \mathscr{R}$, for every $A \in \mathscr{R}\}$. Take the space $\mathscr{M}\left(\mathscr{R}^{\text {loc }}\right)$ of measurable real functions on $\left(\Omega, \mathscr{R}^{\text {loc }}\right)$ and denote by $\mathcal{S}\left(\mathscr{R}^{\text {loc }}\right)$ and $\mathcal{S}(\mathscr{R})$ the space of simple functions with support in $\mathscr{R}^{\text {loc }}$ and $\mathscr{R}$, respectively.

Consider also $v: \mathscr{R} \rightarrow X$ a vector measure, that is, a set function from the $\delta$-ring $\mathscr{R}$ to a real Banach space $X$ for which $\nu\left(\cup_{n} A_{n}\right)=\sum_{n} \nu\left(A_{n}\right)$ in the norm topology of $X$ for all sequences $\left(A_{n}\right)$ of pairwise disjoint members of $\mathscr{R}$ such that $\cup_{n} A_{n} \in \mathscr{R}$. Recall the definition of the semivariation of $\nu$ which is given by $\|\nu\|: \mathscr{R}^{\text {loc }} \rightarrow[0, \infty]$ with $\|\nu\|(A)=$ $\sup \left\{\left|x^{*} \nu\right|(A): x^{*} \in B_{X^{*}}\right\}$ for all $A \in \mathscr{R}^{\text {loc }}$, where $X^{*}$ denotes the topological dual of $X, B_{X^{*}}$ denotes the unit ball in $X^{*}$, and $\left|x^{*} v\right|$ is the usual variation of scalar measure $x^{*} v: \mathscr{R} \rightarrow \mathbb{R}$. The semivariation of $v$ is monotone increasing, countably subadditive, finite on $\mathscr{R}$, and satisfying for all $A \in \mathscr{R}^{\text {loc }}$,

$$
\frac{1}{2}\|\nu\|(A) \leq \sup \left\{\|\nu(B)\|_{X}: B \in \mathscr{R} \cap 2^{A}\right\} \leq\|\nu\|(A) .
$$

We will say that a set $B \in \mathscr{R}^{\text {loc }}$ is $\nu$-null if $\|\nu\|(B)=0$ and that a property holds $\nu$-almost everywhere ( $\nu$-a.e.) if it holds except on a $\nu$-null set. Also, a vector measure $v: \mathscr{R} \rightarrow E$ with values in a Banach lattice $E$ is positive if $\nu(A) \geq 0$ for all $A \in \mathscr{R}$.

Taking into account the previous definitions, the integrability with respect to a vector measure is defined as follows. First, consider the space $L_{w}^{1}(\nu)$ of functions in $\mathscr{M}\left(\mathscr{R}^{\text {loc }}\right)$ such that

$$
\int_{\Omega} f d\left|x^{*} v\right|<+\infty
$$

for all $x^{*} \in X^{*}$ (i.e., which are integrable with respect to $\left|x^{*} \nu\right|$ for all $\left.x^{*} \in X^{*}\right)$, where functions which are equal $\nu$-a.e. are identified. Endow this space with the norm

$$
\|f\|_{\nu}=\sup _{x^{*} \in B_{X^{*}}} \int_{\Omega}|f| d\left|x^{*} v\right|
$$

and the $\nu$-a.e. pointwise order. Then $L_{w}^{1}(\nu)$ is a Banach lattice containing $\delta(\mathscr{R})$ in which convergence in norm of a sequence implies $\nu$-a.e. convergence of some subsequence (see [18, Lemma 3.13]). Even more, the space $L_{w}^{1}(\nu)$ is an ideal of measurable functions in the sense that, if $|f| \leq|g| v$-a.e. with $f \in \mathscr{M}\left(\mathscr{R}^{\text {loc }}\right)$ and $g \in L_{w}^{1}(\nu)$, it follows that $f \in L_{w}^{1}(\nu)$.

Now, take the closed ideal $L^{1}(\nu)$ in $L_{w}^{1}(\nu)$ of functions which are integrable with respect to $v$, that is, the functions in $L_{w}^{1}(\nu)$ for which there exists a vector denoted by $\int_{A} f d v \in X$, such that

$$
x^{*}\left(\int_{A} f d \nu\right)=\int_{A} f d x^{*} \nu \quad \forall x^{*} \in X^{*},
$$

for each $A \in \mathscr{R}^{\text {loc }}$. Then $L^{1}(\nu)$ is a Banach lattice with the norm and the order inherited from $L_{w}^{1}(\nu)$ in which $\delta(\mathscr{R})$ is dense. The space $L^{1}(\nu)$ is also an ideal of measurable functions, and the space $L^{1}(\nu)$ coincides with $L_{w}^{1}(\nu)$ whenever $X$ does not contain any linear subspace isomorphic to $c_{0}[16$, Theorem 5.1].

Regarding the lattice structure of these spaces, recall that $L_{w}^{1}(\nu)$ has the $\sigma$-Fatou property, $L^{1}(\nu)$ is order continuous, $L_{w}^{1}(\nu)_{a}=L_{w}^{1}(\nu)_{a n}=L^{1}(\nu)$, and $L^{1}(\nu)$ is always order dense in $L_{w}^{1}(\nu)$.

Finally, recall the integration operator $I_{\nu}: L^{1}(\nu) \rightarrow X$ given by $I_{\nu}(f)=\int_{\Omega} f d v$ which is linear and continuous with $\left\|I_{\nu}(f)\right\|_{X} \leq\|f\|_{\nu}$.

\section{Representation of Functionals on Spaces of Integrable Functions with respect to a Vector Measure on a $\delta$-Ring}

Let $\mathscr{R}$ be a $\delta$-ring and $v: \mathscr{R} \rightarrow X$ be a vector measure. Following [21, Section 2], we say that a countably additive 
measure $\lambda: \mathscr{R} \rightarrow[0, \infty]$ is a local control measure for $v$ if it satisfies the following conditions:

(1) $\lim _{A \subset B, \lambda(A) \rightarrow 0}\|\nu(A)\|_{X}=0$, for every $B \in \mathscr{R}$,

(2) every $\nu$-null set in $\mathscr{R}^{\text {loc }}$ is $\lambda$-null.

The first condition is equivalent to $\nu(A)=0$ whenever $A \in \mathscr{R}$ with $\lambda(A)=0$ (see [18, Proposition 3.6]), and this happens if and only if every $\lambda$-null set in $\mathscr{R}$ is $\nu$-null, due to the properties of the semivariation. Consequently, the countably additive measure $\lambda$ is a local control measure for $\nu$ if and only if $\lambda$ has the same null sets as $\nu$. From [21, Theorem 3.2], there always exists such a measure, so $L_{w}^{1}(\nu)$ and $L^{1}(\nu)$ are Banach functions spaces related to $\lambda$. Here, by a Banach function space related to $\lambda$, we mean a Banach space $X \subset L^{0}(\lambda)$ satisfying that, if $|f| \leq|g|$ with $f \in L^{0}(\lambda)$ and $g \in X$ then $f \in X$ and $\|f\| \leq\|g\|$, where $L^{0}(\lambda)$ denotes the vector lattice of all measurable real functions on $\Omega$ (functions which are equal $\lambda$ a.e. are identified) endowed with the $\lambda$-a.e. pointwise order.

In the proof of [21, Theorem 3.2], it is shown that there exists a maximal family $\left\{A_{\alpha}\right\}_{\alpha \in \Delta}$ of nonnull sets members of $\mathscr{R}$ satisfying that $A_{\alpha} \cap A_{\beta}$ is $\nu$-null for $\alpha \neq \beta$. Considering that, for each $\alpha \in \Delta$ the class of sets $\mathscr{R} \cap A_{\alpha}:=\{A \in \mathscr{R}$ : $\left.A \subset A_{\alpha}\right\}$, we have that $\mathscr{R} \cap A_{\alpha}$ is a $\delta$-ring as a collection of subsets of $\Omega$ and a $\sigma$-algebra as a collection of subsets of $A_{\alpha}$. Taking $v_{\alpha}$ the restriction of $\nu$ to $\mathscr{R} \cap A_{\alpha}$, it is known that there always exists a finite local control measure $\lambda_{\alpha}$ (called a Rybakov control measure and of type $\left.\left|x^{*} v_{\alpha}\right|\right)$ for $\nu_{\alpha}$. For this measure and for each $B \in \mathscr{R}$, it is proved that $\lambda_{\alpha}\left(A_{\alpha} \cap B\right)=0$ for all $\alpha \in \Delta$ except on a countable set. Now, the set function $\lambda: \mathscr{R} \rightarrow[0, \infty]$ given by $\lambda(A)=\sum_{\alpha \in \Delta} \lambda_{\alpha}\left(A \cap A_{\alpha}\right)$ is a local control measure for $\nu$.

We start our study looking for a suitable definition for the Köthe dual space of $L^{1}(\nu)$. Not only the existence of a local control measure $\lambda$ for $\nu$ but also its particular construction will be the key of our work. Firstly, we establish a useful representation of functionals on spaces $L^{1}(\nu)$. So, let $\mathscr{R}$ be a $\delta$-ring and consider a (countably additive) vector measure $v: \mathscr{R} \rightarrow X$. Fix a decomposition $\left\{A_{\alpha}\right\}_{\alpha \in \Delta}$ of the measure space as the one explained previously and take a countable family $\left\{A_{\alpha_{n}}\right\}_{n \in \mathbb{N}}$ of elements of $\left\{A_{\alpha}\right\}_{\alpha \in \Delta}$. In order to avoid misunderstandings, we advise that, throughout all the paper, the families $\left\{A_{\alpha_{n}}\right\}_{n \in \mathbb{N}}$ are supposed to be $\nu$-a.e. disjoint; that is, all the indexes $\alpha_{n}$ are supposed to be different. Let $\lambda$ be a local control measure for $v$ with the properties of the ones constructed by Brooks and Dinculeanu. Since by the construction $\lambda$ is finite in all $A \in \mathscr{R}$, we have that it is $\sigma$-finite in $C:=\bigcup_{n \in \mathbb{N}} A_{\alpha_{n}} \in \mathscr{R}^{\text {loc }}$.

Lemma 1. Let $\left\{A_{\alpha_{n}}\right\}_{n \in \mathbb{N}}$ in $\left\{A_{\alpha}\right\}_{\alpha \in \Delta}$ and $C:=\bigcup_{n \in \mathbb{N}} A_{\alpha_{n}}$ as explained above. Then there is a finite measure $\lambda_{C}: \mathscr{R}^{\text {loc }} \cap$ $C \rightarrow \mathbb{R}$ such that, for each $\varphi \in\left(L^{1}(\nu)\right)^{*}$, there is a measurable function $g_{\varphi, C} \in L^{0}\left(\lambda_{C}\right)$ such that

$$
\varphi(f)=\int_{C} f g_{\varphi, C} d \lambda_{C}
$$

for all $f \in L^{1}(\nu)$ so that $\operatorname{supp}\{f\} \subseteq C$.
Proof. Let $\lambda$ be a fixed local control measure for $v$ constructed as explained previously. For each $n \in \mathbb{N}$, consider the measure $\lambda_{n}: \mathscr{R} \cap C \rightarrow \mathbb{R}$ given by $\lambda_{n}:=\left.\lambda\right|_{A_{\alpha_{n}}}$. Note that all these measures are finite by the construction of $\lambda$. Consider the finite measure $\lambda_{C}: \mathscr{R}^{\text {loc }} \cap C \rightarrow \mathbb{R}$ given by $\lambda_{C}(B):=\sum_{n=1}^{\infty}\left(\lambda_{n}\left(A_{\alpha_{n}} \cap B\right) / 2^{n} \lambda_{n}\left(A_{\alpha_{n}}\right)\right), B \in \mathscr{R}^{\text {loc }} \cap C$.

Define also the finite measure $\lambda_{\varphi, C}: \mathscr{R}^{\text {loc }} \cap C \rightarrow \mathbb{R}$ by $\lambda_{\varphi, C}(B):=\sum_{n=1}^{\infty}\left(\varphi\left(\chi_{A_{\alpha_{n}} \cap B}\right) / 2^{n}\|\nu\|\left(A_{\alpha_{n}}\right)\right), B \in \mathscr{R}^{\text {loc }} \cap C$. Note that this measure is well defined and absolutely continuous with respect to $\lambda_{C}: \mathscr{R}^{\text {loc }} \cap C \rightarrow \mathbb{R}$. By the Radon-Nikodym theorem, there is an integrable function $g_{\varphi, C}^{0} \in L^{1}\left(\lambda_{C}\right)$ such that

$$
\lambda_{\varphi, C}(B)=\int_{B} g_{\varphi, C}^{0} d \lambda_{C}, \quad B \in \mathscr{R}^{\text {loc }} \cap C .
$$

Thus, for each $f \in \mathcal{S}(\mathscr{R})$, we have that

$$
\int f d \lambda_{\varphi, C}=\int_{B} f g_{\varphi, C}^{0} d \lambda_{C}
$$

Take the function $h_{C}:=\sum_{n=1}^{\infty} 2^{n}\|\nu\|\left(A_{\alpha_{n}}\right) \chi_{A_{\alpha_{n}}}$, and note that, for such an $\mathscr{R}$-simple function $f$, we have that

$$
\begin{aligned}
\int_{C} f h_{C} d \lambda_{\varphi, C} \\
=\int_{C} f\left(\sum_{n=1}^{\infty} 2^{n}\|\nu\|\left(A_{\alpha_{n}}\right) \chi_{A_{\alpha_{n}}}\right) d \lambda_{\varphi, C}=\varphi\left(f \chi_{C}\right) .
\end{aligned}
$$

Thus, for such a function $f$ we obtain that

$$
\varphi(f)=\int_{C} f h_{C} d \lambda_{\varphi, C}=\int_{C} f h_{C} g_{\varphi, C}^{0} d \lambda_{C} .
$$

Write $g_{\varphi, C}:=h_{C} g_{\varphi, C}^{0}$. A direct calculation using the order continuity of $L^{1}(\nu)$ gives the result for this function $g_{\varphi, C}$.

(1) Let us consider now the following construction in order to define the Köthe dual of $L^{1}(\nu)$. Fix again a maximal $\mathscr{R}$-decomposition $\left\{A_{\alpha}\right\}_{\alpha \in \Delta}$ and $\lambda: \mathscr{R} \rightarrow[0, \infty]$ a local control measure for $\nu$. For every countable family $\left\{A_{\alpha_{n}}\right\}_{n \in \mathbb{N}}$ in $\left\{A_{\alpha}\right\}_{\alpha \in \Delta}$, we can define the following: the set $C=\bigcup_{n} A_{\alpha_{n}}$, a $\delta$-ring $\mathscr{R}_{C}$ of subsets of $C$ by $\mathscr{R}_{C}:=\{A \in \mathscr{R}: A \subseteq C\}$, and a countably additive positive measure $\hat{\lambda}_{C}:\left(\mathscr{R}_{C}\right)^{\text {loc }} \rightarrow[0, \infty]$ given by $\widehat{\lambda}_{C}(A)=\sum_{n} \lambda\left(A \cap A_{\alpha_{n}}\right), A \in\left(\mathscr{R}_{C}\right)^{\text {loc }}$.

Notice that the measure $\hat{\lambda}_{C}$ is well defined; although the measure $\lambda$ is defined just for elements of $\mathscr{R}$, using classical arguments, it can be immediately seen that this formula extends it to the whole $\left(\mathscr{R}_{C}\right)^{\text {loc }}$.

Note also that $\mathscr{R}_{\mathrm{C}}=\mathscr{R} \cap C$ and remark that $\left(\mathscr{R}_{\mathrm{C}}\right)^{\text {loc }}$ is computed with respect to the target space $C$ that is, $\left(\mathscr{R}_{C}\right)^{\text {loc }}:=$ $\left\{A \subseteq C: A \cap B \in \mathscr{R}_{C}, B \in \mathscr{R}_{C}\right\}$. In order to clarify the relation between the measurability with respect to the different $\sigma$-algebras involved, let us establish the following notation. If $B$ is a subset of $\mathscr{R}^{\text {loc }}$, we write $\left(\mathscr{R}_{B}\right)^{\text {loc }_{B}}$ for the $\sigma$ algebra associated with $\mathscr{R}_{B}$ using as target space the set $B$. No explicit reference is made when $B=\Omega$. The relation between $\left(\mathscr{R}_{C}\right)^{{ }^{\text {loc }} \text { C }}$ and $\mathscr{R}^{\text {loc }}$ is now done by the following lemma. The proof is straightforward. 
Lemma 2. Let $C=\bigcup_{n=1}^{\infty} A_{\alpha_{n}}$. Then $\left(\mathscr{R}_{C}\right)^{\text {loc }}=\mathscr{R}^{\text {loc }} \cap C$. In particular, the measurable functions in $\mathscr{M}\left(\Omega, \mathscr{R}^{\text {loc }}\right)$ when restricted to $C$ are measurable with respect to $\left(C,\left(\mathscr{R}_{C}\right)^{\text {loc } C}\right)$, and $L^{0}(\lambda)$ when the functions are restricted to $C$ coincides with $L^{0}\left(\left.\lambda\right|_{C}\right)$.

For a given function $f \in L^{1}(\nu)$, we can find a countable set $\left\{\alpha_{n}\right\}_{n \in \mathbb{N}}$ of different elements of $\Delta$ such that the support of $f$ outside $C_{f}=\bigcup_{n} A_{\alpha_{n}}$ is $\nu$-a.e. null (and so $|\lambda|\left(\Omega \backslash C_{f}\right)=0$; see [20, Theorem 3.6]), and we can consider the $\sigma$-finite measure $\widehat{\lambda}_{f}:=\widehat{\lambda}_{C_{f}}$. Therefore, for a given function $f \in L^{1}(\nu)$, we find a set $C_{f}$, a $\delta$-ring $\mathscr{R}_{C_{f}}$, and a $\sigma$-finite measure $\hat{\lambda}_{f}$.

We describe now the adequate family of functions-like objects necessary to define the Köthe dual of an abstract space $L^{1}(\nu)$. For $\alpha \in \Delta$, define the sets $L_{\alpha}^{0}(\lambda)$ of classes of $\lambda$-a.e. equal $\mathscr{R} \cap A_{\alpha}$-measurable functions in $\mathbb{R}^{A_{\alpha}}$. Consider the Cartesian product $\prod_{\alpha \in \Delta} L_{\alpha}^{0}(\lambda)$. For each countable set $C$ of subsets $A_{\alpha_{n}}$, consider $g_{C}$ an element of $\prod_{\alpha \in \Delta} L_{\alpha}^{0}(\lambda)$ that is a measurable function in each $A_{\alpha_{n}}$ and 0 out of $C$. Take the vector space $\mathscr{F}_{0}$ - with the obvious sum and product-of the families $G:=\left\{g_{C}\right\}$ of elements $g_{C} \in \prod_{\alpha \in \Delta} L_{\alpha}^{0}(\lambda)$ that are compatible; that is, for each couple of countable families $C$ and $C^{\prime}$, if there is an $\alpha \in \Delta$ such that $A_{\alpha}$ belongs both to $C$ and $C^{\prime}$, then the respective " $\alpha$-coordinates" $\left(g_{C}\right)_{A_{\alpha}}$ and $\left(g_{C^{\prime}}\right)_{A_{\alpha}}$ coincide $\left.\lambda\right|_{A_{\alpha}}$-a.e. A natural norm-type function to consider for the elements of this space is given by

$\|G\|_{\mathscr{F}}$

$$
:=\sup \left\{\left.\int_{C} g_{C} f\right|_{C} d \widehat{\lambda}_{C}: f \in B_{L^{1}(v)},\right.
$$

$$
\left.C \text { countable union of } A_{\alpha}^{\prime} s\right\}, \quad G \in \mathscr{F}_{0} \text {. }
$$

It can be easily seen that this expression is a norm for the space $\mathscr{F}$ of all the elements of $\mathscr{F}_{0}$ for which $\|\cdot\|_{\mathscr{F}}$ is finite. This construction allows to write the extension of the definition of Köthe dual for the setting of the abstract $L^{1}(\nu)$ spaces as $\left(\mathscr{F},\|\cdot\|_{\mathscr{F}}\right)$; that is, we define

$$
\begin{aligned}
& \left(L^{1}(\nu)\right)^{\prime} \\
& :=\left\{G \in \mathscr{F}:\left.g_{C_{f}} \cdot f\right|_{C_{f}} \in L^{1}\left(C_{f},\left(\mathscr{R}_{C_{f}}\right)^{\operatorname{loc}_{C_{f}}}, \widehat{\lambda}_{f}\right),\right. \\
& \left.\quad \forall f \in L^{1}(\nu)\right\},
\end{aligned}
$$

with the dual norm $\|G\|_{\left(L^{1}(\nu)\right)^{\prime}}:=\|G\|_{\mathscr{F}}$.

(2) In what follows, we will consider a slightly different context in order to obtain a better representation of the Köthe dual space defined as in previous. Using the fixed decomposition given by $\left\{A_{\alpha}\right\}_{\alpha \in \Delta}$, define the $\delta$-ring $\mathscr{R}_{0}$ of the elements $A=\bigcup_{n=1}^{k} A_{n}, A_{n} \in \mathscr{R}$ such that there is an $\alpha_{n} \in \Delta$ with $A_{n} \subseteq A_{\alpha_{n}}, k \in \mathbb{N}$. Note that $\mathscr{R}_{0} \subseteq \mathscr{R}$ and so we have that $\mathscr{R}^{\text {loc }} \subseteq\left(\mathscr{R}_{0}\right)^{\text {loc }}$. We can then define the measure $\bar{\lambda}: \mathscr{R}_{0} \rightarrow \mathbb{R}$ given by $\bar{\lambda}(A):=\sum_{n=1}^{k} \lambda\left(A_{n}\right)$, where $A \in \mathscr{R}_{0}$ is decomposed as mentioned earlier. The variation $|\bar{\lambda}|$ of this measure is well defined and allows its extension to $\left(\mathscr{R}_{0}\right)^{\text {loc }}$. Note also that $\mathscr{M}\left(\mathscr{R}^{\text {loc }}\right) \subseteq \mathscr{M}\left(\mathscr{R}_{0}\right)^{\text {loc }}$.

Proposition 3. For a vector measure $v$, consider a maximal $\mathscr{R}$-decomposition $\left\{A_{\alpha}\right\}_{\alpha \in \Delta}$. Then

$$
\begin{aligned}
& \left(L^{1}(\nu)\right)^{\prime} \\
& :=\left\{G \in \mathscr{F}: g_{C} \cdot f \in L^{1}\left(\Omega,\left(\mathscr{R}_{0}\right)^{\text {loc }},|\bar{\lambda}|\right),\right. \\
& \left.\quad \forall f \in L^{1}(\nu)\right\},
\end{aligned}
$$

and the norm for this space can be computed by

$$
\begin{aligned}
\|G\|_{\mathscr{F}} & :=\sup _{f \in B_{L^{1}(v)}} \int_{C_{f}} g_{C_{f}} f d|\bar{\lambda}| \\
& =\sup \left\{\int_{C} g_{C} f d|\bar{\lambda}|: f \in B_{L^{1}(v)}, C=\bigcup_{n=1}^{\infty} A_{\alpha_{n}}\right\} .
\end{aligned}
$$

Proof. It is a consequence of the following equivalences. Let $C$ be a countable union of elements $A_{\alpha_{n}}$. If $G \in \mathscr{F}$ and $f \in L^{1}(\nu)$ such that $\operatorname{supp}\{f\} \subseteq C$, the function $f \cdot g_{C_{f}}$ can be supposed to be 0 outside the set $C_{f}$, and so $g_{C} f=g_{C_{f}} f$. Then $f \cdot g_{C_{f}} \in$ $L^{1}\left(\widehat{\lambda}_{f}\right)$ if and only if

$$
\int f g_{C_{f}} d \widehat{\lambda}_{f}=\sum_{n=1}^{\infty} \int_{A_{\alpha_{n}}} f g_{C_{f}} d \widehat{\lambda}_{f}<\infty
$$

Write $\lambda_{n}$ for the measure $\lambda_{\alpha_{n}}(\cdot)=\lambda\left(\cdot \cap A_{\alpha_{n}}\right)$, where $A_{\alpha_{n}} \in C_{f}$, and note that

$$
\begin{aligned}
\int f g_{C_{f}} d \widehat{\lambda}_{f} & =\sum_{n=1}^{\infty} \int_{A_{\alpha_{n}}} f g_{C_{f}} d \lambda_{n} \\
& =\sum_{n=1}^{\infty} \int_{A_{\alpha_{n}}} f g_{C_{f}} d|\bar{\lambda}|=\int f g_{C_{f}} d|\bar{\lambda}| \\
& =\int_{C} f g_{C} d|\bar{\lambda}| .
\end{aligned}
$$

Therefore, integrability of $\left.f g_{C_{f}}\right|_{C_{f}}$ with respect to $\hat{\lambda}_{f}$ is equivalent to integrability of $f g_{C}$ with respect to $|\bar{\lambda}|$, and the proof is complete.

Theorem 4. For a vector measure $v,\left(L^{1}(v)\right)^{*}=\left(L^{1}(v)\right)^{\prime}$ isometrically, where $\left(L^{1}(v)\right)^{\prime}$ is defined from any maximal $\mathscr{R}$ decomposition $\left\{A_{\alpha}\right\}_{\alpha \in \Delta}$.

Proof. It is a consequence of Lemma 1, and Proposition 3. Let us show the proof in two steps.

Step 1. Let $\varphi \in\left(L^{1}(\nu)\right)^{*}$ and $C=\bigcup_{n=1}^{\infty} A_{\alpha_{n}}$. Then using Lemma 1 we find a function $g_{\varphi, C}$ representing the RadonNikodym derivative of the set function $\varphi\left(\left.\cdot\right|_{C}\right)$-that is a measure by the order continuity of $L^{1}(\nu)$-in a way that

$$
\varphi(f)=\int_{C} f g_{\varphi, C} d \lambda_{C}
$$


for all $f \in L^{1}(\nu)$ such that $\operatorname{supp}\{f\} \subseteq C$. Therefore, computing in each $C$ the Radon-Nikodym derivative $\tau_{C}$ of the measure $\lambda_{C}$ appearing in Lemma 1 , with respect to $\hat{\lambda}_{C}$, and writing

$$
\begin{aligned}
\varphi(f) & =\int_{C} f g_{\varphi, C} d \lambda_{C} \\
& =\int_{C} f g_{\varphi, C} \tau_{C} d \widehat{\lambda}_{C}=\int_{C} f g_{\varphi, C} \tau_{C} d|\bar{\lambda}|
\end{aligned}
$$

whenever $\operatorname{supp}\{f\} \subseteq C$, we obtain a family $G$ of the space $\mathscr{F}_{0}$. Moreover, note that for such $G$, we have that

$$
\begin{aligned}
& \|G\|_{\mathscr{F}} \\
& =\sup \left\{\left|\int_{C} g_{C} f d \widehat{\lambda}_{C}\right| \leq\|\varphi\|: f \in B_{L^{1}(v)}, C=\bigcup_{n=1}^{\infty} A_{\alpha_{n}}\right\},
\end{aligned}
$$

and so $G \in \mathscr{F}$. In fact, it can be easily seen that the identification $\varphi \leadsto G$ is linear, continuous, and injective.

Step 2. Let us show now that this identification is also surjective. We use Proposition 3. Let $G$ be a compatible family of elements of $\mathscr{F}_{0}$ such that $\|G\|_{\mathscr{F}}<\infty$. For each $f \in L^{1}(\nu)$, define

$$
\varphi(f)=\int_{C_{f}} f g_{C_{f}} d|\bar{\lambda}|
$$

and notice that, for each countable union $C$ of sets $A_{\alpha_{n}}, \alpha_{n} \in$ $\Delta$, such that $C_{f} \subseteq C$,

$$
\int_{C_{f}} f g_{C_{f}} d|\bar{\lambda}|=\int_{C} f g_{C} d|\bar{\lambda}|
$$

as a consequence of the compatibility of the elements of $\mathscr{F}_{0}$ that define the family $G$. This allows to prove that $\varphi$ is well defined and linear; the finiteness of $\|G\|_{\mathscr{F}}$ gives also that $\sup _{f \in B_{L^{1}(\nu)}}|\varphi(f)| \leq\|G\|_{\mathscr{F}}<\infty$, and so $\varphi$ is an element of the dual space $\left(L^{1}(\nu)\right)^{*}$.

Thus, we have proved that $\left(\mathscr{F},\|\cdot\|_{\mathscr{F}}\right)=$ $\left(\left(L^{1}(\nu)\right)^{*},\|\cdot\|_{\left(L^{1}(v)\right)^{*}}\right)$ isometrically.

Remark 5. Notice that $\left(L^{1}(\nu)\right)^{\prime}$ is a Banach lattice with the order of $\left(L^{1}(\nu)\right)^{*}$. It can be easily seen that it coincides with the natural order in $\mathscr{F}_{0}$.

Remark 6. Note that, for the case that the $\delta$-ring $\mathscr{R}$ is a countable union of $\sigma$-algebras (i.e., the $\sigma$-finite case), a better representation for the Köthe dual is possible. Instead of defining it as the space of families of countable supported elements of a Cartesian product, a single function can play the role; that is,

$$
\begin{aligned}
& \left(L^{1}(\nu)\right)^{\prime} \\
& :=\left\{g \in L^{0}(\lambda):\left.g \cdot f\right|_{C_{f}} \in L^{1}\left(C_{f},\left(\mathscr{R}_{C_{f}}\right)^{\left.\operatorname{loc}_{C_{f}}, \hat{\lambda}_{f}\right),}\right.\right. \\
& \left.\quad \forall f \in L^{1}(\nu)\right\} .
\end{aligned}
$$

This is a trivial consequence of the definition of the families, since in the $\sigma$-finite case just a function defines a family. Taking into account the definition of the measures involved in the expression above, it is clear that this representation equals

$$
\begin{aligned}
& \left(L^{1}(\nu)\right)^{\prime} \\
& \quad:=\left\{g \in L^{0}(\lambda): g \cdot f \in L^{1}(\lambda), \forall f \in L^{1}(\nu)\right\},
\end{aligned}
$$

that is the well-known definition of Köthe dual for the $\sigma$-finite case.

We extend now the definition of the Köthe dual to abstract Banach lattices. Let $E$ be an order continuous Banach lattice. Then it is possible to define its Köthe dual by means of an integral representation (using vector measures over $\delta$-rings) which can be described as follows. First, by [12, Proposition 1.a.9], E can be decomposed into an unconditionally direct sum of a family of mutually disjoints ideals $\left\{E_{\alpha}\right\}_{\alpha \in \Delta}$, each $E_{\alpha}$ having a weak unit. Each $E_{\alpha}$ is now an order continuous Banach lattice with a weak unit and so, from [22, Theorem 8], there exist a $\sigma$-algebra $\Sigma_{\alpha}$ of parts of an abstract set $\Omega_{\alpha}$ and a positive vector measure $\nu_{\alpha}: \Sigma_{\alpha} \rightarrow E_{\alpha}$ such that the integration operator $I_{v_{\alpha}}: L^{1}\left(v_{\alpha}\right) \rightarrow E_{\alpha}$ is an order isometry. Consider the set $\Omega=\bigcup_{\alpha \in \Delta}\{\alpha\} \times \Omega_{\alpha}$ and the $\delta$-ring $\mathscr{R}$ of subsets of $\Omega$ given by the sets $\bigcup_{\alpha \in \Delta}\{\alpha\} \times A_{\alpha}$ satisfying that $A_{\alpha} \in \Sigma_{\alpha}$ for all $\alpha \in \Delta$ and there exists a finite set $I \subset \Delta$ such that $A_{\alpha}$ is $\nu_{\alpha}$-null for all $\alpha \in \Delta \backslash I$. Then, take the vector measure $v: \mathscr{R} \rightarrow E$ defined by

$$
\nu\left(\bigcup_{\alpha \in \Delta}\{\alpha\} \times A_{\alpha}\right)=\sum_{\alpha \in \Delta} v_{\alpha}\left(A_{\alpha}\right)
$$

and compute the integration operator $I_{v}: L^{1}(\nu) \rightarrow E$. This operator is an order isometry (see [1, Theorem 4$]$ and [3, pp. $22-23])$. Let $\left(\Omega,\left(\mathscr{R}_{0}\right)^{\text {loc }},|\bar{\lambda}|\right)$ be the measure space associated with $\nu$. The definition of the Köthe dual of $E$ is done using this integral representation adequately:

$$
\begin{aligned}
E^{\prime} & =\left(L^{1}(\nu)\right)^{\prime} \\
& =\left\{G \in \mathscr{F}: g_{C} \cdot I_{\nu}^{-1}(e) \in L^{1}\left(\Omega,\left(\mathscr{R}_{0}\right)^{\mathrm{loc}},|\bar{\lambda}|\right), \forall e \in E\right\} .
\end{aligned}
$$

Corollary 7. Let E be an order continuous Banach lattice. Let $v$ be the vector measure which appears in the representation explained previously. Then $E^{*}=E^{\prime}$.

Let us prove now a related result regarding the Köthe dual of $L_{w}^{1}(\nu)$. The representation of the Köthe dual of $L^{1}(\nu)$ given in Proposition 3 suggests the following definition of Köthe dual space for any Banach lattice $L$ of $|\bar{\lambda}|$-a.e. classes of $\left(\Omega,\left(\mathscr{R}_{0}\right)^{\text {loc }}\right)$-measurable functions, in particular, for $L_{w}^{1}(\nu)$. We define the Köthe dual of $L$ as

$$
L^{\prime}:=\left\{G \in \mathscr{F}: g_{C} \cdot f \in L^{1}\left(\Omega,\left(\mathscr{R}_{0}\right)^{\text {loc }},|\bar{\lambda}|\right), \forall f \in L\right\},
$$


endowed with the norm

$$
\|G\|_{L^{\prime}}:=\sup _{f \in B_{L}} \int_{C} g_{C} f d|\bar{\lambda}| .
$$

Let us show that, as in the finite measure case-that is, in the case of a vector measure on a $\sigma$-algebra-, the Köthe duals of $L^{1}(\nu)$ and $L_{w}^{1}(\nu)$ coincide. For a Banach function space on a $\sigma$ finite measure space, this is the well-known fact that $\left(X_{a}\right)^{*}=$ $\left(X_{a}\right)^{\prime}=X^{\prime}$-where $X_{a}$ is the order continuous part of $X$ whenever $X_{a}$ is super order dense in $X$.

Corollary 8. Let $v$ be a vector measure on a $\delta$-ring. Then one has that $\left(L_{w}^{1}(\nu)\right)^{\prime}=\left(L^{1}(\nu)\right)^{\prime}=L^{1}(\nu)^{*}$.

Proof. The inclusion $\left(L_{w}^{1}(\nu)\right)^{\prime} \subseteq\left(L^{1}(\nu)\right)^{\prime}$ is obvious. If $G \in$ $L^{1}(\nu)^{\prime}$, for each countable family $C$, we have that $\left.g_{C} f\right|_{C} \in$ $L^{1}(|\bar{\lambda}|)$ for each $f \in L^{1}(\nu)$.

Consider the restriction of $L^{1}(\nu)$ to functions with support in $C$. For every function $0 \leq h \in L_{w}^{1}(\nu)$ with support in $C$, we have that there is an increasing sequence $0 \leq s_{n}$ in $S(\mathscr{R})$ converging a.e. to $h$ (see the proof of [2, Theorem 4.8]). Notice also that, for all $n$,

$$
\left\|s_{n}\right\|_{L^{1}(v)} \leq\|h\|_{L_{w}^{1}(v)}<\infty
$$

If $h \in L_{w}^{1}(\nu)$, we have that the restriction $\mid g_{C} h \|_{C}$ can be approximated $|\bar{\lambda}|$-a.e. using an increasing sequence $s_{n}$ of simple functions with support in $C$ as previously mentioned: $\left|g_{C}\right| s_{n}$ increases a.e. to $\left|g_{C} h\right|$ where the sequence is clearly in $L^{1}(|\bar{\lambda}|)$. The monotone convergence theorem when applied to the sequence $\left(\left|g_{C}\right| s_{n}\right)_{n}$ of $L^{1}(|\bar{\lambda}|)$ gives that $\left.g_{C} h\right|_{C} \in L^{1}(|\bar{\lambda}|)$. Theorem 4 completes the proof.

Corollary 9. Let $v$ be a vector measure on $a \delta$-ring and consider $L$ an ideal of $L_{w}^{1}(\nu)$ containing $L^{1}(\nu)$. Then $L^{\prime}=$ $\left(L^{1}(\nu)\right)^{\prime}$.

Let $E$ be a Banach lattice with the Fatou property having its $\sigma$-order continuous part $E_{a}$ as an order dense subset. As explained for the order continuous case, it is again possible to define its Köthe dual using a suitable representation for $E$ by means of a space of integrable functions. Let us describe briefly the elements we need. Consider the vector measure $v$ associated with $E_{a n}=E_{a}$ as in Corollary 7, then $I_{v}: L^{1}(\nu) \rightarrow$ $E_{a n}$ is an order isometry. Extend now $I_{\nu}$ to the space $L_{w}^{1}(\nu)$ as follows. For every $0 \leq f \in L_{w}^{1}(\nu)$, choose $\left(\varphi_{n}\right) \subset \mathcal{S}\left(\mathscr{R}^{\text {loc }}\right)$ such that $0 \leq \varphi_{n} \uparrow f$. For each $n$ and $I \subset \Delta$ finite, take $\xi_{(n, I)}=$ $\varphi_{n} \chi_{\bigcup_{\alpha \in I}\{\alpha\} \times \Omega_{\alpha}} \in \mathcal{S}(\mathscr{R})$ and consider $e=\sup _{(n, I)} I_{\nu}\left(\xi_{(n, I)}\right)$ in $E$. Finally, define $T_{\nu}(f)=e$ and extend to the general case. The extension $T_{v}$ defined in this way is again an order isometry between $L_{w}^{1}(\nu)$ and $E$ (see [1, Theorem 8]). Consequently, the definition of the Köthe dual of $E$ can be done using this representation; that is,

$$
\begin{aligned}
E^{\prime} & =\left(L_{w}^{1}(\nu)\right)^{\prime} \\
& =\left\{G \in \mathscr{F}: g_{C} \cdot T_{\nu}^{-1}(e) \in L^{1}\left(\Omega,\left(\mathscr{R}_{0}\right)^{\text {loc }},|\bar{\lambda}|\right), \forall e \in E\right\},
\end{aligned}
$$

where $\left(\Omega,\left(\mathscr{R}_{0}\right)^{\text {loc }},|\bar{\lambda}|\right)$ is the measure space associated with $\nu$.

Corollary 10. Let $E$ be a Banach lattice with the Fatou property having its $\sigma$-order continuous part $E_{a}$ as an order dense subset. Let $v$ be the vector measure which appears in the representation explained previously. Then $\left(E_{a}\right)^{*}=\left(E_{a}\right)^{\prime}=E^{\prime}$.

\section{Some Examples and Applications}

(1) Consider $1 \leq p<\infty$ and the space $\ell^{p}(\Gamma)$ for a noncountable index set $\Gamma$. It is an order continuous Banach lattice. Consider the $\delta$-ring $\mathscr{R}_{\Gamma}$ defined by the finite subsets of $\Gamma$ and the vector measure $v_{p}: \mathscr{R}_{\Gamma} \rightarrow \ell^{p}(\Gamma)$. Then the space $L^{1}\left(\nu_{p}\right)$ can be directly computed and coincides with $\ell^{p}(\Gamma)$ isometrically and in order (see example 2.2 in [20] and example 4.1 and the comments before Theorem 5.1. in [2]). Fixing the maximal decomposition of $\Gamma$ given by the singletons $A_{\gamma}:=\{\gamma\}, \gamma \in \Gamma$ and after Proposition 3, we get that its Köthe dual space is given by

$$
\left(L^{1}\left(v_{p}\right)\right)^{\prime}:=\left\{G \in \mathscr{F}: g_{C} \cdot\left(\omega_{\Gamma}\right) \in \ell^{1}(\Gamma), \forall\left(\omega_{\Gamma}\right) \in \ell^{p}(\Gamma)\right\},
$$

since $L^{1}\left(\Gamma,\left(\mathscr{R}_{\Gamma}\right)^{\text {loc }}, \eta\right)$ is in this case $\ell^{1}(\Gamma)$, taking into account that the variation of the local control measure $\lambda$ in this case can be taken to be the counting measure and also that obviously $\mathscr{R}_{0}=\mathscr{R}_{\Gamma}$. Since the maximal decomposition that we are considering is given by all the singletons, we can identify each element $G \in \mathscr{F}$ with an element of $\mathbb{R}^{\Gamma}$, and so $\left(L^{1}(\nu)\right)^{\prime}$ can be written as

$$
\left(L^{1}\left(\nu_{p}\right)\right)^{\prime}:=\left\{\left(\eta_{\gamma}\right):\left(\eta_{\gamma}\right) \cdot\left(\omega_{\Gamma}\right) \in \ell^{1}(\Gamma), \forall\left(\omega_{\Gamma}\right) \in \ell^{p}(\Gamma)\right\} .
$$

This characterization and Theorem 4 give that the dual space coincides with this Köthe dual space. This description has been frequently used in examples and computations of general Banach space theory. The reader can find these arguments for instance in [23] (see Remark 2.1), where the computation of the Köthe dual is based on the fact that the related measure space is discrete.

The same construction can be done for the case when the vector measure defined previously takes its values in $\ell^{\infty}(\Gamma)$. Then it is known that $L^{1}\left(\nu_{\infty}\right)=c_{0}(\Gamma)$ and $L_{w}^{1}\left(\nu_{\infty}\right)=\ell^{\infty}(\Gamma)$. Thus the (Banach) lattice $\ell_{0}^{\infty}(\Gamma)$ of the elements of $\ell^{\infty}(\Gamma)$ with countable support satisfies $c_{0}(\Gamma) \subseteq \ell_{0}^{\infty}(\Gamma) \subseteq \ell^{\infty}(\Gamma)$. The constructive arguments given previously for $c_{0}(\Gamma)$ instead of $\ell^{p}(\Gamma)$ together with Corollaries 8 and 9 give that all the spaces appearing in the inclusion $c_{0}(\Gamma) \subseteq \ell_{0}^{\infty}(\Gamma) \subseteq \ell^{\infty}(\Gamma)$ satisfy that 
their Köthe duals coincide and are equal to $\ell^{1}(\Gamma)$, providing in this way a description of the Köthe dual of the relevant space $\ell_{0}^{\infty}(\Gamma)$

(2) Although the construction of (1) regards discrete vector measures, the results in this paper can also be applied for general Banach lattices of functions involving measures that are not discrete. Let us write the Köthe dual of the space appearing in Remark 22 of [4]. Consider a family of disjoint probability spaces $\left(\Omega_{\gamma}, \Sigma_{\gamma}, \mu_{\gamma}\right)_{\gamma \in \Gamma}$, where $\Gamma$ is an uncountable set of indexes. The $\delta$-ring $\mathscr{R}$ is defined by finite unions $B=$ $\bigcup_{i=1}^{n} A_{\gamma_{i}}, A_{\gamma_{i}} \in \Sigma_{\gamma_{i}}$. We consider the vector measure $\kappa: \mathscr{R} \rightarrow$ $c_{0}(\Gamma)$ given by $\kappa(B)=\sum_{i=1}^{n} \mu_{\gamma_{i}}\left(A_{\gamma_{i}}\right) \chi_{\left\{\gamma_{i}\right\}}$. As in example 2.2 of [20], it can be easily shown that the space $L^{1}(\kappa)$ is the direct sum $\bigoplus_{c_{0}(\Gamma)} L^{1}\left(\mu_{\gamma}\right)$. Consequently, the support of an element of this space is contained in a countable union of sets $\Omega_{\gamma}$, $\gamma \in \Gamma$. However, $L_{w}^{1}(\kappa)=\bigoplus_{\ell^{\infty}(\Gamma)} L^{1}\left(\mu_{\gamma}\right)$ and the functions of this space can be even strictly positive in all points of $\bigcup_{\gamma \in \Gamma} \Omega_{\gamma}$. This is suggested by the symbols $\bigoplus_{c_{0}(\Gamma)}$ and $\bigoplus_{\ell^{\infty}(\Gamma)}$ that we use: in the first case, countability of the support of each element is assumed but not in the second. Thus, we can describe an element $f \in L^{1}(\kappa)$ as a sequence $\left(f_{\gamma_{i}}\right)_{i=1}^{\infty}$, where each $f_{\gamma_{i}} \in L^{1}\left(\mu_{\gamma_{i}}\right)$. Let us consider the variation $|\eta|$ of the local control measure $\eta$ for $\kappa$ that is defined for each element $B=\bigcup_{i=1}^{n} A_{\gamma_{i}} \in \mathscr{R}$ as $\eta(B)=\sum_{i=1}^{n} \mu_{\gamma_{i}}\left(A_{\gamma_{i}}\right)$. The Köthe dual of $L^{1}(\kappa)$ is then given by

$$
\begin{aligned}
& \left(L^{1}(\kappa)\right)^{\prime} \\
& \quad:=\left\{G \in \mathscr{F}: g_{C} \cdot f \in L^{1}\left(\Omega,\left(\mathscr{R}_{0}\right)^{\mathrm{loc}},|\eta|\right), \forall f \in L^{1}(\kappa)\right\},
\end{aligned}
$$

that can also be written as

$$
\begin{aligned}
& \left(L^{1}(\kappa)\right)^{\prime} \\
& =\left\{G \in \mathscr{F}: g_{C} \cdot\left(f_{\gamma_{i}}\right)_{i=1}^{\infty} \in L^{1}\left(\Omega,(\mathscr{R})^{\mathrm{loc}},|\eta|\right),\right. \\
& \left.\bigcup_{i=1}^{n} A_{\gamma_{i}} \subseteq C, f \in L^{1}(\kappa)\right\},
\end{aligned}
$$

where each $g_{C}$ of an element $G$ of $\mathscr{F}$ can be identified with an element of the direct sum $\bigoplus_{\ell^{1}(\Gamma)} L^{\infty}\left(\mu_{\gamma}\right)$ in the usual manner. Note that, in this case, $\mathscr{R}$ and $\mathscr{R}_{0}$ coincide. After Theorem 4, we know that this provides a description of the dual space $\left(L^{1}(\kappa)\right)^{*}$. By Corollary 8 , this space coincides also with $\left(L_{w}^{1}(\kappa)\right)^{\prime}$.

\section{Acknowledgments}

M. A. Juan was supported by Ministerio de Economía y Competitividad (Spain) (Project MTM2011-23164). E. Jiménez Fernández was supported by Junta de Andalucía and FEDER Grant (P09-FQM-4911) (Spain) and by Ministerio de Economía y Competitividad (Spain) (Project MTM201236740-C02-02). E. A. Sánchez-Pérez was supported by Ministerio de Economía y Competitividad (Spain) (Project MTM2012-36740-C02-02).

\section{References}

[1] O. Delgado and M. A. Juan, "Representation of Banach lattices as $L_{w}^{1}$ spaces of a vector measure defined on a $\delta$-ring," Bulletin of the Belgian Mathematical Society, vol. 19, no. 2, pp. 239-256, 2012.

[2] J. M. Calabuig, O. Delgado, M. A. Juan, and E. A. Sánchez Pérez, "On the Banach lattice structure of $L_{w}^{1}$ of a vector measure on a $\delta$-ring, Collectanea Mathematica," 2013.

[3] G. P. Curbera, El espacio de funciones integrables respecto de una medida vectorial [Ph.D. thesis], Universidad de Sevilla, 1992.

[4] J. M. Calabuig, M. A. Juan, and E. A. Sánchez Pérez, "Spaces of $p$-integrable functions with respect to a vector measure defined on a $\delta$-ring," Operators and Matrices, vol. 6, no. 2, pp. 241-262, 2012.

[5] G. P. Curbera and W. J. Ricker, "The Fatou property in $p$ convex Banach lattices," Journal of Mathematical Analysis and Applications, vol. 328, no. 1, pp. 287-294, 2007.

[6] S. Okada, W. J. Ricker, and E. A. Sánchez Pérez, Optimal Domain and Integral Extension of Operators Acting in Function Spaces, vol. 180 of Operator Theory: Advances and Applications, Birkhäuser, Basel, Switzerland, 2008.

[7] I. Ferrando and E. A. Sánchez Pérez, "Tensor product representation of the (Pre)dual of the $L_{p}$-space of a vector measure," Journal of the Australian Mathematical Society, vol. 87, no. 2, pp. 211-225, 2009.

[8] F. Galaz-Fontes, "The dual space of $L_{p}$ of a vector measure," Positivity, vol. 14, no. 4, pp. 715-729, 2010.

[9] E. A. Sánchez Pérez, "Vector measure duality and tensor product representations of $L_{p}$-spaces of vector measures," Proceedings of the American Mathematical Society, vol. 132, no. 11, pp. 3319-3326, 2004.

[10] S. Okada, "The dual space of $L_{1}(\mu)$ for a vector measure $\mu$," Journal of Mathematical Analysis and Applications, vol. 177, no. 2, pp. 583-599, 1993.

[11] C. D. Aliprantis and O. Burkinshaw, Positive Operators, Academic Press, New York, NY, USA, 1985.

[12] J. Lindenstrauss and L. Tzafriri, Classical Banach Spaces II, Springer, Berlin, Gemany, 1979.

[13] W. A. J. Luxemburg and A. C. Zaanen, Riesz Spaces I, NorthHolland, Amsterdam, The Netherlands, 1971.

[14] P. Meyer-Nieberg, Banach Lattices, Springer, New York, NY, USA, 1991.

[15] A. C. Zaanen, Riesz Spaces II, North-Holland, Amsterdam, The Netherlands, 1983.

[16] D. R. Lewis, "On integrability and summability in vector spaces," Illinois Journal of Mathematics, vol. 16, pp. 294-307, 1972.

[17] P. R. Masani and H. Niemi, "The integration theory of Banach space valued measures and the Tonelli-Fubini theorems I. Scalar-valued measures on $\delta$-rings," Advances in Mathematics, vol. 73, no. 2, pp. 204-241, 1989.

[18] P. R. Masani and H. Niemi, "The integration theory of Banach space valued measures and the Tonelli-Fubini theorems. II. Pettis integration," Advances in Mathematics, vol. 75, no. 2, pp. 121-167, 1989.

[19] G. F. Stefansson, " $L_{1}$ of a vector measure," Le Matematiche, vol. 48, no. 2, pp. 219-234, 1993.

[20] O. Delgado, " $L^{1}$-spaces of vector measures defined on $\delta$-rings," Archiv der Mathematik, vol. 84, no. 5, pp. 432-443, 2005. 
[21] J. K. Brooks and N. Dinculeanu, "Strong additivity, absolute continuity and compactness in spaces of measures," Journal of Mathematical Analysis and Applications, vol. 45, no. 1, pp. 156175, 1974.

[22] G. P. Curbera, "Operators into $L^{1}$ of a vector measure and applications to Banach lattices," Mathematische Annalen, vol. 293, no. 1, pp. 317-330, 1992.

[23] H. J. Lee, M. Martín, and J. Merí, "Polynomial numerical indices of Banach spaces with absolute norm," Linear Algebra and Its Applications, vol. 435, no. 2, pp. 400-408, 2011. 


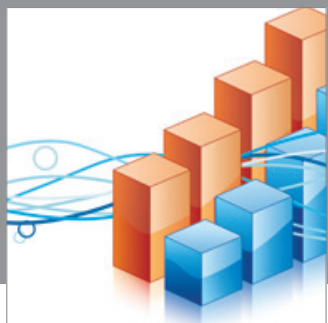

Advances in

Operations Research

mansans

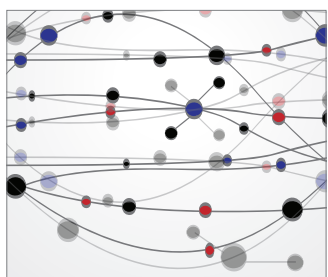

The Scientific World Journal
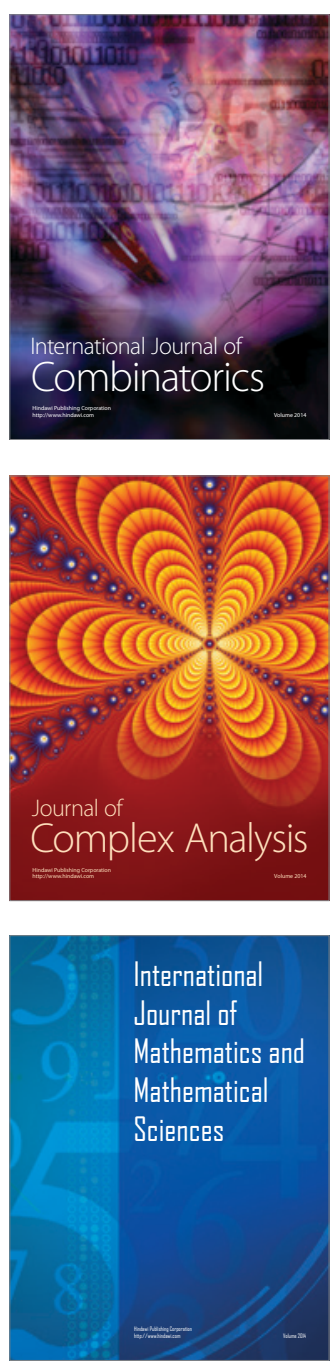
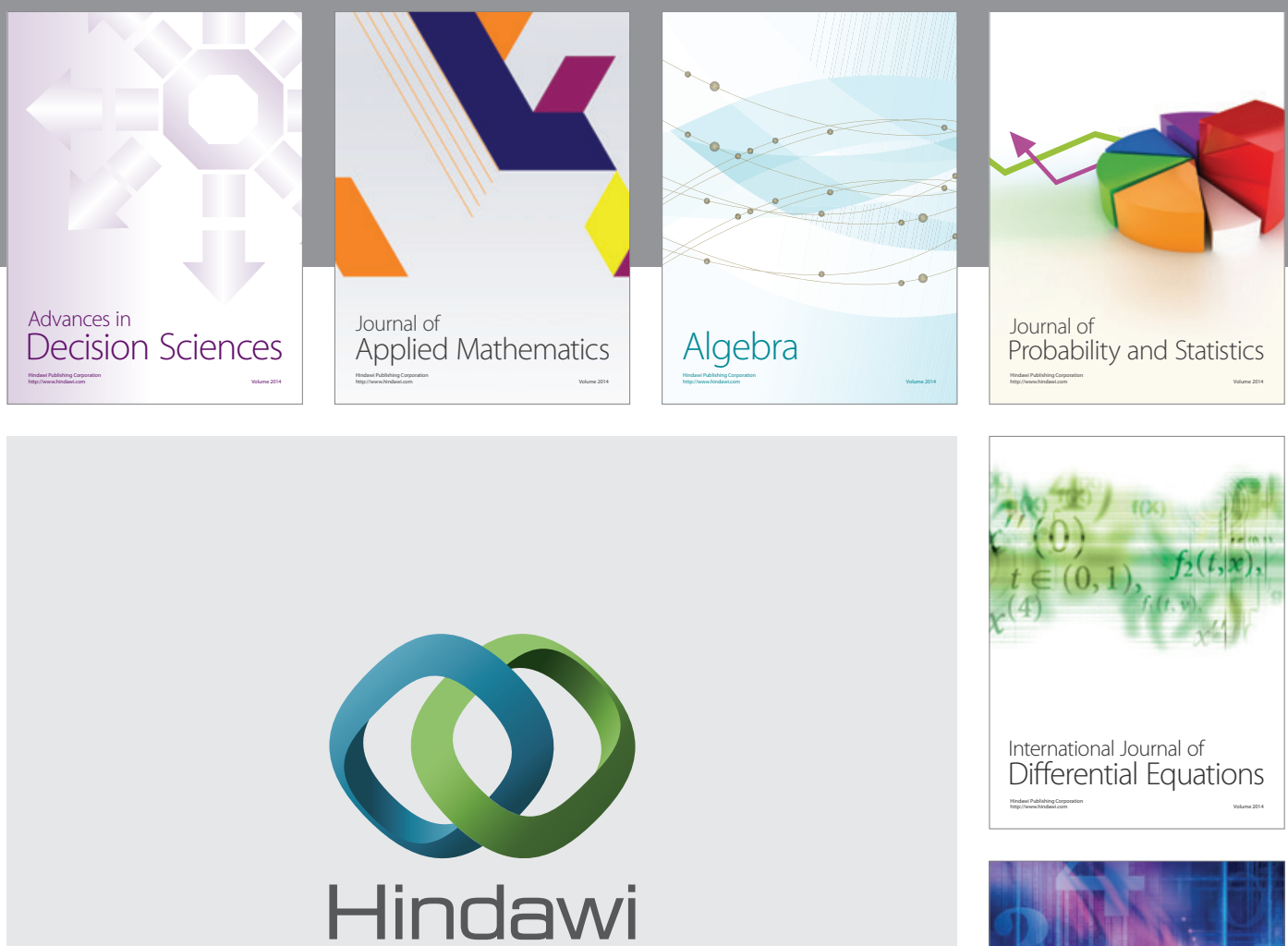

Submit your manuscripts at http://www.hindawi.com
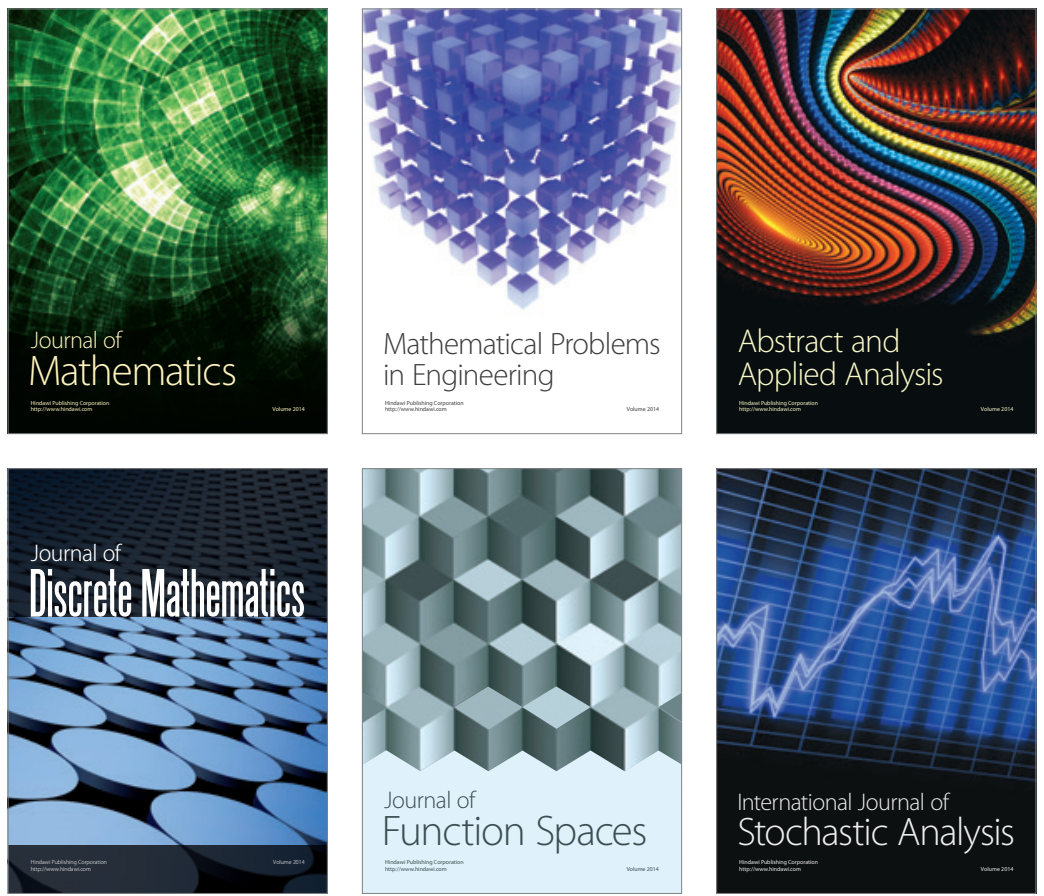

Journal of

Function Spaces

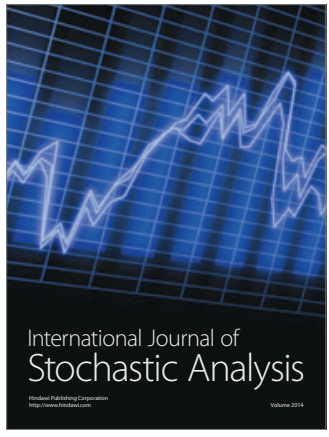

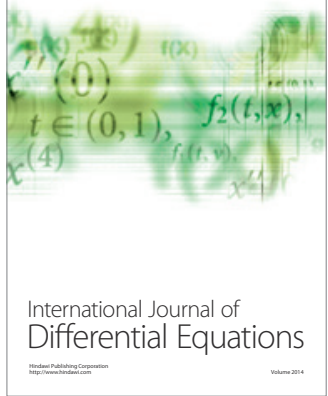
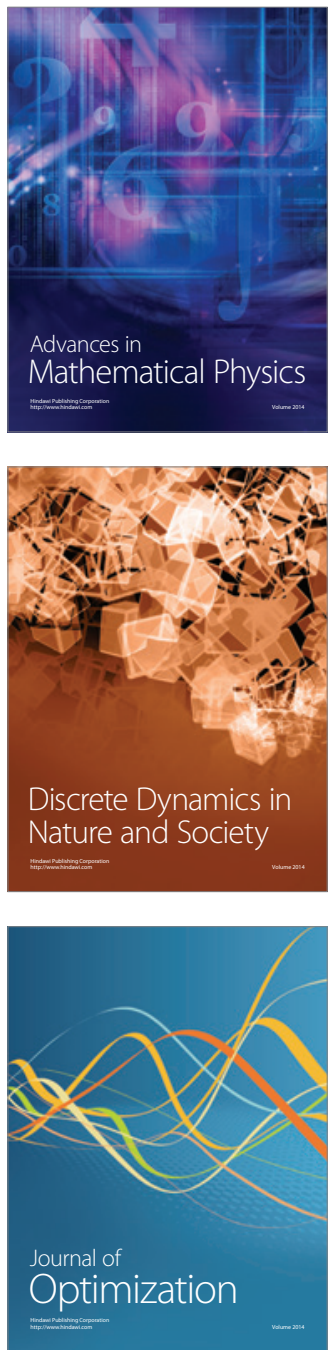